\title{
LOS ARCHIVOS NORTEAMERICANOS COMO RECURSOS PARA LA INVESTIGACIÓN DE LA HISTORIA ARGENTINA DEL SIGLO XX
}

\author{
OS ARQUIVOS NORTE-AMERICANOS COMO RECURSOS PARA A \\ INVESTIGAÇÃO DA HISTÓRIA ARGENTINA DO SÉCULO XX
}

\author{
Daniel Lvovich \\ Universidad Nacional de General Sarmiento - UNGS \\ Consejo Nacional de Investigaciones Científicas y Técnicas- CONICET \\ dlvovich@ungs.edu.ar
}

\section{Resumen}

En este artículo nos proponemos presentar lo que entendemos son los principales usos potenciales de los archivos públicos de los Estados Unidos como recursos para la investigación histórica sobre la Argentina. Con tal fin, presentamos en primer lugar un panorama general sobre el sistema de archivos públicos estadounidense; proponemos en segundo término algunos modos de acceder a la información disponible sobre Argentina en los repositorios norteamericanos. Por último, brindamos una perspectiva panorámica y algunos ejemplos del modo en que se construyó la información sobre Argentina y describimos de manera sucinta aquellos tipos de documentos que a nuestro entender resultan particularmente útiles para el estudio de la historia argentina.

Palabras Claves: Archivos. Estados Unidos. Argentina

\section{Resumo}

Neste artigo propomos apresentar o que entendemos ser os principais usos potenciais dos arquivos públicos dos Estados Unidos como recursos para pesquisa histórica sobre a Argentina. Para tanto, apresentamos em primeiro lugar uma visão geral do sistema de registros públicos americanos, propondo, em segundo lugar, algumas formas de acessar as informações disponíveis sobre a Argentina nos repositórios norte-americanos. Por fim, fornecemos uma perspectiva panorâmica e alguns exemplos da forma como as informações 
sobre a Argentina foram construídas e descrevemos sucintamente os tipos de documentos que, até onde sabemos, são particularmente úteis para o estudo da história argentina.

Palavras-chave: Arquivos. Estados Unidos. Argentina.

\section{1- Introducción}

En este artículo nos proponemos presentar lo que entendemos son los principales usos potenciales de los archivos públicos de los Estados Unidos como recursos para la investigación histórica sobre la Argentina. Con tal fin, presentamos en primer lugar un panorama general sobre el sistema de archivos públicos estadounidense; proponemos en segundo término algunos modos de acceder a la información disponible sobre Argentina en los repositorios norteamericanos. Por último, brindamos una perspectiva panorámica y algunos ejemplos del modo en que se construyó la información sobre Argentina y describimos de manera sucinta aquellos tipos de documentos que a nuestro entender resultan particularmente útiles para el estudio de la historia argentina.

El trabajo se realiza a partir de nuestra experiencia como investigador en los archivos norteamericanos y presenta las ventajas y limitaciones de tal origen, ya que hemos tenido la oportunidad de trabajar con determinadas colecciones documentales, en base a las cuales podemos brindar un panorama del tipo de información existente y de la lógica de su agrupación, pero -por supuesto- no hemos relevado la totalidad de la documentación disponible en esos repositorios.

\section{2- El sistema de archivos públicos de los Estados Unidos}

La conservación de los archivos federales norteamericanos está a cargo de la Administración Nacional de Archivos y Registros (National Archives and Record Administration, NARA). Los NARA tienen dos sedes principales, en Washington DC y en College Park, Maryland, en el área metropolitana de la capital estadounidense.

La sede de Washington, conocida como NARA I, contiene en un museo la exhibición permanente de la Constitución, la Carta de Derechos, y la Declaración de Independencia norteamericanas y conserva documentación en papel y en microfilm concernientes a genealogía. Para los investigadores, el edifico de los Archivos Nacionales en Washington DC 
contiene documentos textuales y en microfilm referentes a genealogía, los nativos americanos, el New Deal, el Distrito de Columbia, las Cortes Federales, el Congreso y asuntos marítimos, navales y militares previos a la Segunda Guerra Mundial. Se puede consultar la información sobre las colecciones conservadas en esta sede en su página web: https://www.archives.gov/dc.

La sede de College Park, conocida como NARA II, conserva en soportes en papel, microfilm o electrónico el archivo de ilustraciones y fotografías, documentos cartográficos y arquitectónicos, pertenecientes a la presidencia de Richard Nixon, documentos relacionados a películas, sonido, y videos, la colección de documentos referentes al asesinato de John F. Kennedy, y documentos textuales pertenecientes a la mayoría de las agencias civiles y documentos militares posteriores a la Segunda Guerra Mundial. La página web de NARA II es https://www.archives.gov/college-park.

El sistema archivístico federal se complementa por un lado con el Registro Federal, con sede en Washington DC (www.archives.gov/federal-register/contact.html) que administra la documentación oficial sobre Leyes Federales, Documentos Presidenciales, Reglas Administrativas y Avisos, Descripciones de Organizaciones Federales, Programas y Actividades, el Colegio Electoral y el proceso de Enmiendas Constitucionales, y por otro lado con doce archivos regionales distribuidos a lo largo de todo el país.

Bajo la órbita de los NARA y con sede en diversos puntos de los Estados Unidos se encuentran las bibliotecas de los presidentes Hoover, Roosevelt, Truman, Eisenhower, Kennedy, Johnson, Nixon, Ford, Carter, Reagan, Bush y Clinton. Estas colecciones ofrecen a la consulta una muy extensa documentación personal y política de la época en que cada uno de ellos ejerció la primera magistratura norteamericana.

Las documentaciones de los presidentes norteamericanos desde fines del siglo XVIII hasta comienzos del siglo XX se conservan en cambio en la sala de manuscritos de la Biblioteca del Congreso de los Estados Unidos. Este mismo repositorio conserva también documentación referida a distintas personalidades públicas, procesos y movimientos políticos y sociales, manuscritos relativos a la Guerra Civil norteamericana, entre otros. Una parte de estos recursos se puede consultar online, incluyendo por ejemplo la documentación de los presidentes George Washington o Abraham Lincoln, y de los generales John Pershing y George Patton y los papeles de Hannah Arendt y Sigmund Freud, entre muchos otros. El catálogo general de la sala de manuscritos de la Biblioteca del Congreso se puede consultar en 
esta dirección: https://www.loc.gov/rr/mss/findaid.html, y la documentación en línea en este vínculo: https://www.loc.gov/rr/mss/ammem.html.

Como ocurre con otros países que han alcanzado roles hegemónicos a nivel mundial y desarrollado políticas imperialistas, los archivos públicos de los Estados Unidos reúnen de manera sistemática información relevante sobre casi todos los países del mundo. La recolección de información a través de una vasta red de diplomáticos, militares, agentes de inteligencia o viajeros generó una enorme masa de información - textual, fotográfica, planigráfica - acerca de las más diversas actividades en casi todos los países del mundo. La sistematización y conservación de esta información es a la vez una de las condiciones necesarias para alcanzar y sostener un rol hegemónico como potencia mundial, y un claro testimonio de ese poderío.

De acuerdo a la temática y a la agencia estatal que los produce, los plazos en que la distinta documentación es puesta a la consulta pública varía. Por supuesto, la documentación perteneciente a la CIA (Central Intelligence Agency o Agencia Central de Inteligencia), el Departamento de Defensa y otras áreas que producen informaciones particularmente sensibles o comprometedoras es la que requiere mayor tiempo antes de ser librada a la consulta pública.

Existen sin embargo maneras de acceder a una parte de esta información a través de las disposiciones de la llamada Ley de Libertad de Información conocida como FOIA (Siglas de Freedom of Information Act, https://www.foia.gov/), que permite a los ciudadanos por un lado pedir información personal y por otro solicitar que determinados documentos sean revisados para su desclasificación. Debido a esta ley, cada agencia del gobierno norteamericano tiene una oficina especializada en el tema que revisa los documentos y decide si estos pueden ser completamente desclasificados, parcialmente desclasificados, pero con algunas partes censuradas, o si deben permanecer secretos. La FOIA posee varias disposiciones que permiten al gobierno estadunidense continuar guardando con carácter de secreto ciertos documentos, si el revisor estima que la desclasificación de determinada información puede comprometer la seguridad nacional de los Estados Unidos.

Una institución que ha abogado y logrado la desclasificación de archivos es la National Security Archives (NSA), de la Universidad George Washington. La NSA ha logrado la desclasificación de miles de documentos y en su página web se puede acceder por suscripción a más de cien mil documentos desclasificados, correspondientes al período que se extiende entre las presidencias de Truman y Obama, y organizados en colecciones referidas a 
políticas presidenciales, temas nucleares, Derechos Humanos, Inteligencia, militares, Guerra Fría, Asia, América Latina y Medio Oriente (https://nsarchive.gwu.edu/digital-nationalsecurity-archive). En ocasiones, la documentación se publica en libros impresos y sirve de base a investigaciones, como en el célebre caso de Pinochet: los archivos secretos editado por Peter Kornbluh (2004), que da cuenta de la intervención directa del gobierno de Estado Unidos en el golpe de Pinochet de 1973.

Sin embargo, también existe el procedimiento inverso, y se han retirado de la consulta documentos previamente desclasificados por considerarse que su publicidad afecta la seguridad nacional norteamericana. Un ejemplo de la información sobre estos procedimientos de reclasificación se puede observar en este listado remitido por la NARA's Public Affairs Office: https://www.archives.gov/files/about/plans-reports/withdrawn/2016withdrawals.pdf.

\section{3- El acceso a la información sobre Argentina en los Archivos Nacionales de los Estados Unidos}

Los National Archives tienen un sistema de clasificación de la información complejo, por lo que la manera de acceder a la misma no resulta siempre sencilla. Existen por tanto diversas colecciones y distintos criterios para acceder a la información disponible sobre la Argentina. En este apartado brindaremos algunos ejemplos al respecto, sin la pretensión de aportar una guía completa y exhaustiva.

Una manera de acceder a la información reunida sobre la Argentina es través de los fondos organizados por país (Country files), agencias y épocas. Por ejemplo, se pueden consultar los 24 rollos de microfilm que reúnen la información sobre la Argentina producida por el Departamento de Estado entre 1910 y 1929, y clasificados temáticamente en categorías como asuntos internos, economía, sociedad, relaciones con EEUU, relaciones con otros países, entre otros. También se encuentran disponibles, solo para brindar otro ejemplo, veinte cajas de Archivo con la información en papel sobre la Argentina correspondiente al período comprendido entre 1953 y 1971, compilada por la US Information Agency y organizada según diversas temáticas de la vida política, económica y social argentina.

Otro modo de acceso es a través de fondos sobre temas específicos que contienen entre otros muchos casos- información sobre la Argentina. Tal es el caso, entre otros, del fondo especial sobre el Holocausto, que contiene materiales -en rollos de microfilms o en 
cajas de archivos en papel- específicamente dedicados a la Argentina, con información proveniente de agencias civiles y en particular del Departamento de Estado (https://www.archives.gov/research/holocaust/finding-aid/civilian/rg-59-5.html), y de agencias militares y de inteligencia (https://www.archives.gov/research/holocaust/findingaid/civilian/rg-59-5.html). Pertenece a un caso similar -dentro del registro de los bienes y servicios militares provistos por Estados Unidos a terceros países desde la década de 1950los 1.920 documentos referidos a la provisión de asistencia militar a la Argentina entre las décadas de 1960 y 1990, cuyo catálogo se puede consultar en línea en la siguiente dirección: https://aad.archives.gov/aad/display-partial-records.jsp?f=3285\&mtch=1920\&q=argentina\&c at $=\mathrm{TS} 15 \& \mathrm{dt}=822 \& \mathrm{tf}=\mathrm{F} \& \mathrm{bc}=\mathrm{s} 10 \& \mathrm{rpp}=50 \& \mathrm{pg}=38$

De particular interés para la investigación sobre la historia reciente argentina y sobre todo para la historia de la última dictadura argentina resultan un conjunto de materiales, que además cuentan con la inestimable ventaja de estar total o parcialmente disponibles en línea. Nos referimos, por un lado, a los Central Foreign Policy Files, que reúnen más de 3.200.000 telegramas y cables que dan cuenta de la política exterior norteamericana entre 1973 y 1979. Entre ellos, el investigador puede acceder por internet a más de 20.000 telegramas referidos a la Argentina en dicho período y al catálogo de unos 3.300 cables que se pueden consultar en papel en NARA II. El sitio en que se accede a esta información es el siguiente: https://aad.archives.gov/aad/free-text-search-results.jsp?cat=WR43\&bc=sl\&q=argentina\&btn Search $=$ Search\&as_alq $=\& a s \_a n q=\& a s \_e p q=\& a s \_w o q=$

Por otro lado, se encuentran disponibles también en línea un amplio conjunto de documentos desclasificados reunidos en colecciones (Chile, Guatemala, Argentina, El Salvador, E-Mails de Hillary Rodham Clinton en su función de Secretaria de Estado y transcripciones de las conversaciones telefónicas de Henry Kissinger), publicados por el Departamento de Estado en aplicación de la Ley de Libertad de Información (FOIA). En ese conjunto, se inscriben 4.903 documentos desclasificados referidos a la Argentina, a los que se accede en esta dirección web: https://foia.state.gov/Search/Results.aspx?collection=ARGENTINA\&searchText=*.

Como señalamos, también se pueden consultar documentos desclasificados en la página web de los National Security Archives, institución que además ha realizado tareas de cooperación con otras organizaciones con el fin de cruzar y complementar la información obrante en archivos norteamericanos con los de otros países. Un ejemplo al respecto es el 
Proyecto Timerman, que articula los NSA con el Archivo de la Dirección de Inteligencia de la Provincia de Buenos Aires, dependiente de la Comisión Provincial por la Memoria (https://nsarchive2.gwu.edu/NSAEBB/NSAEBB299/index.htm).

Al momento de escribirse estas líneas, se espera que se efectivice una nueva desclasificación de documentos relativos a la última dictadura argentina, según se anunció en Washington y en Buenos Aires el 24 de marzo de 2019.

\section{4- La construcción de la información disponible: una perspectiva panorámica y algunos ejemplos}

Distintas agencias estatales estadounidenses participaban de la construcción del conocimiento que sobre la Argentina -al igual que sobre otros países del mundo- disponía el gobierno norteamericano. Dichas agencias podían tener nivel ministerial -como por ejemplo los Departamentos de Estado, de Defensa, de Comercio o de Agricultura- o ser entes de inteligencia, como la OSS o la CIA ${ }^{\mathrm{i}}$, entre otras. Las fuentes de la información resultan amplísimas: publicaciones y estadísticas oficiales del gobierno argentino, prensa comercial y política, información política resultado de los análisis realizados por funcionarios destinados en la embajada en Buenos Aires o por agentes de inteligencia, registro de reuniones oficiales o de conversaciones con funcionarios nacionales y provinciales de distinto nivel, así como con representantes de la vida política, sindical, empresarial, cultural, eclesiástica, militar y social de la Argentina -que en algunas ocasiones preferían preservar su anonimato en los registros documentales-. También cartas y peticiones presentadas por ciudadanos argentinos al gobierno norteamericano, o comunicaciones de ciudadanos norteamericanos al ejecutivo o a miembros del poder legislativo de su país referidas a personas o situaciones que se desarrollaban en la Argentina, entre muchas otras.

Todo ello brindaba al gobierno norteamericano una masa de informaciones muy amplia sobre distintos aspectos de la vida argentina, en base a la cual se definían estrategias, desarrollaban políticas y realizaban previsiones.

Por supuesto, la multiplicidad de agencias involucradas, las diferencias de perspectivas y opiniones de los actores participantes y las diversas fuentes a las que recurrían para construir su información determinaban que la información disponible no resultara siempre homogénea y en ocasiones fuera contradictoria. 
Un buen ejemplo es el brindado por las interpretaciones de la Semana Trágica de 1919. En sus comunicaciones al Departamento de Estado de enero y de febrero de 1919, el Embajador estadounidense en Argentina Frederic Jesup Stimson mostraba su convicción de que los acontecimientos de enero se habían visto instigados tanto por Alemania -interesada en afectar a los aliados en el momento de la firma del armisticio y el desarrollo de los tratados de Paz- como por la Rusia soviética. El embajador señalaba la responsabilidad de Hipólito Irigoyen en los acontecimientos, dada lo que consideraba una débil actitud frente al movimiento obrero, pero estaba convencido de encontrarse frente a un complot maximalista. Stimson señalaba como una prueba de la existencia de dicho complot que de los 182 muertos en lo que definía como los "primeros combates del levantamiento", 150 de los cadáveres recogidos por las autoridades en las calles correspondían a judíos rusos ${ }^{\mathrm{ii}}$. Ese mismo día, en un telegrama a su gobierno, el embajador transmitía las estimaciones oficiales que calculaban las bajas entre los manifestantes en 500 muertos y 550 heridos, pero dos semanas después, en una comunicación del 27 de enero, informaba al Departamento de Estado que el número de muertos en el "levantamiento" era de $1.356 \mathrm{y}$ el de heridos de alrededor de 5.000 iii.

En contraste, fuentes de inteligencia militar, sin descartar una eventual instigación bolchevique a lo que era considerado no ya un levantamiento sino un estallido, estimaban las víctimas en 800 muertos y 2000 heridos, incluyendo muchos "inocentes",iv . Pocas semanas más tarde, el boletín semanal de inteligencia que recibía el presidente Wilson y las más altas autoridades norteamericanas, mostraba lo poco fundado del rumor que atribuía a los rusos la responsabilidad en la agitación de enero, apelando para ello a las cifras acerca de la nacionalidad de los más importantes dirigentes "maximalistas" de la Argentina, entre los cuales los originarios de Rusia eran solo 8 en un total de $116^{\mathrm{v}}$. 


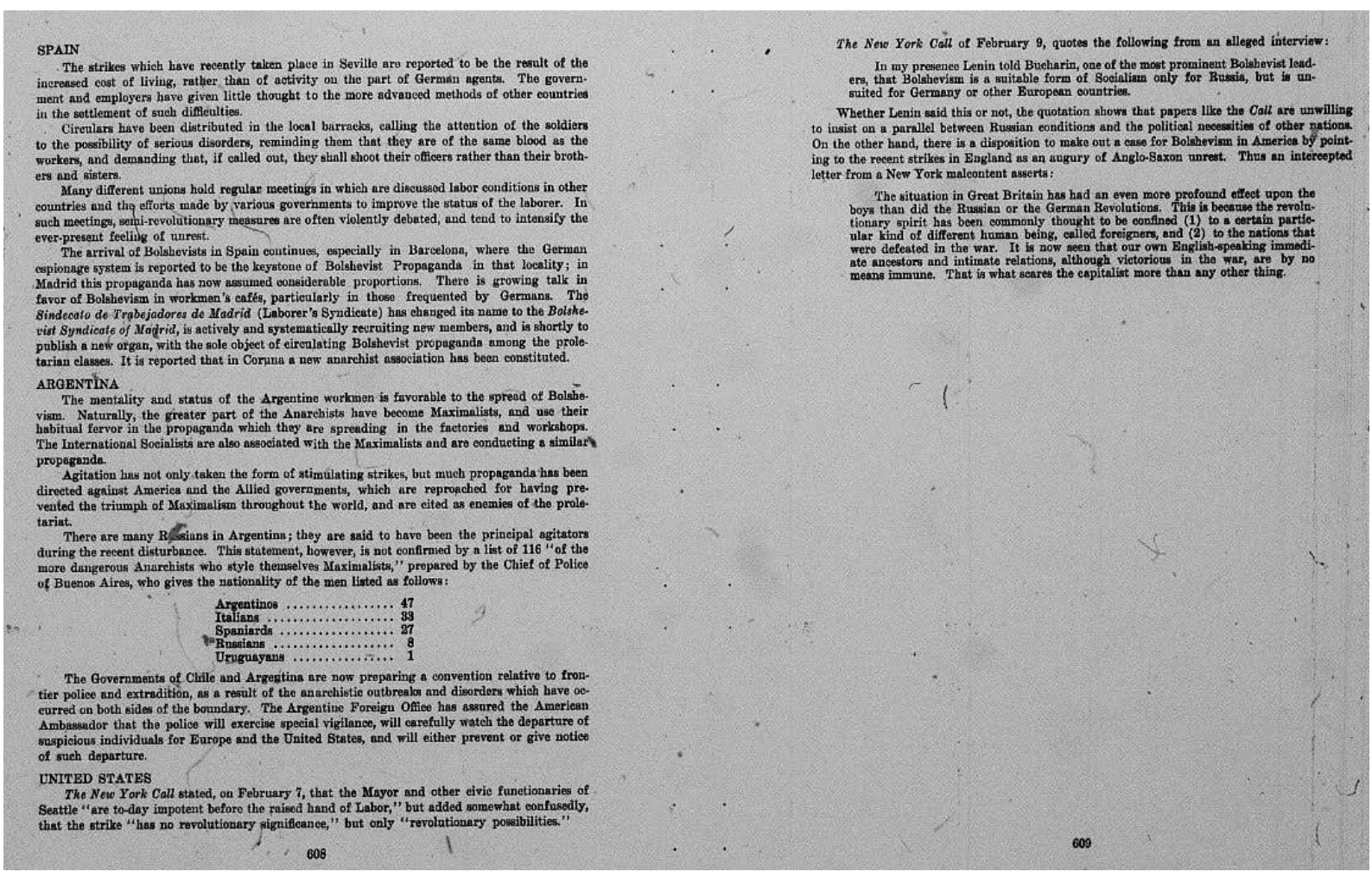

Reproducción de una página del sumario semanal de inteligencia, 22 de febrero de 1919

Fuente: Fotografía del autor

Respecto a las fuentes en que se basaban las informaciones, encontramos allí una variedad gigantesca. Entre la enorme cantidad de ejemplos disponibles, preferimos presentar dos muy diferenciados.

En un extremo, tenemos la transcripción de la conversación sostenida en febrero de 1971 entre el embajador norteamericano y el general Roberto Marcelo Levingston, por entonces a cargo de la presidencia de facto, en la que éste manifestó su alineamiento con las políticas norteamericanas, dio garantías para la inversión extranjera y manifestó una posición marcadamente anticomunista y su preocupación por los procesos políticos de Chile, Bolivia y Uruguay. Se trata de un documento confidencial donde el presidente de facto daba cuenta, con una franqueza muy difícil de sostener en un discurso público, de sus posiciones ${ }^{\mathrm{vi}}$. 


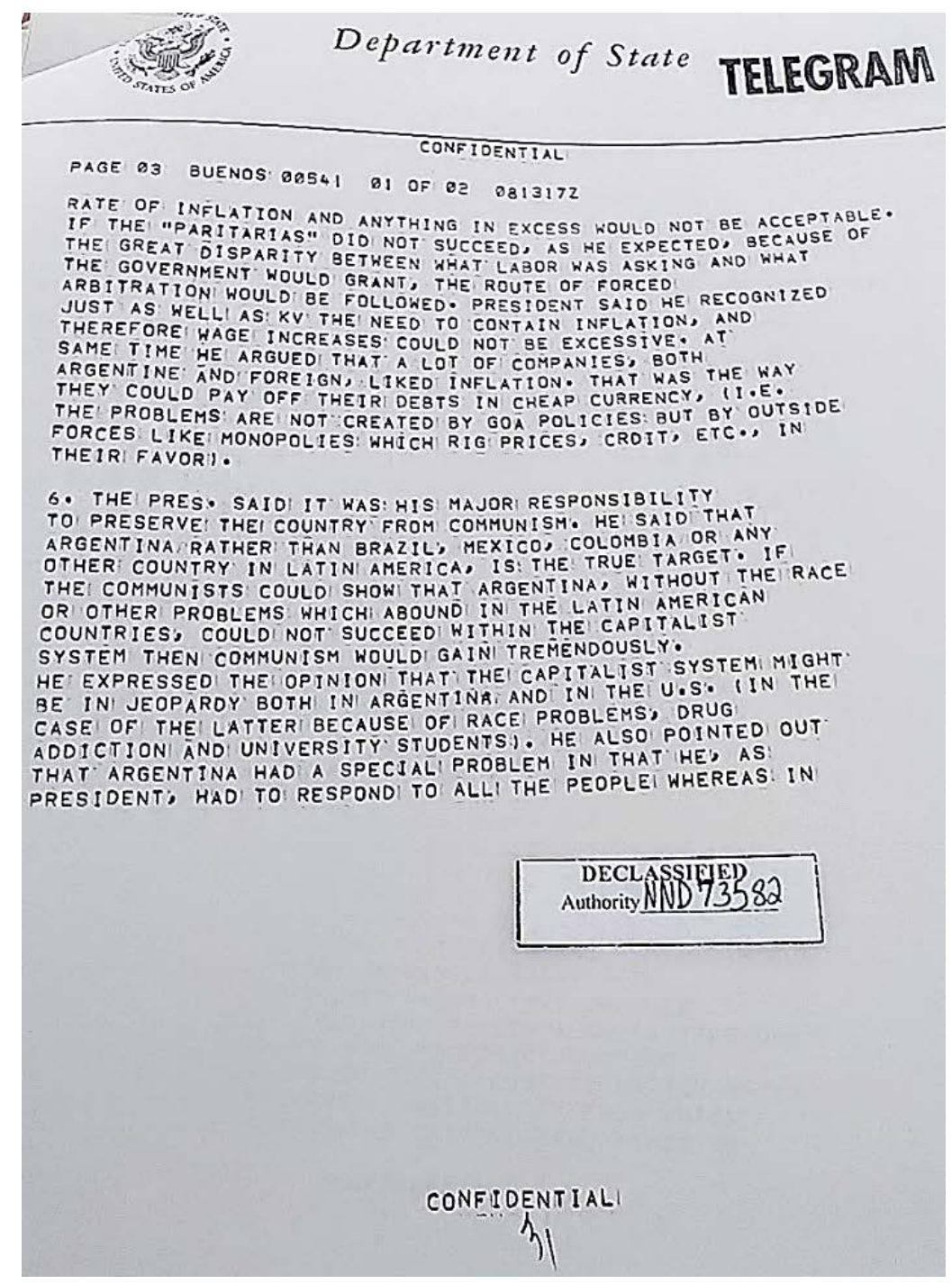

Reproducción de una página del telegrama que contiene las minutas de la conversación entre el embajador norteamericano y el presidente de facto Levingston

Fuente: Fotografía del autor

En el otro extremo, presentamos como ejemplo el caso de un anónimo militante del Partido Comunista Argentino (PCA) que informó a la inteligencia norteamericana acerca del desarrollo de un acto del Encuentro Nacional de los Argentinos en junio de 1971 y de la perspectiva de la dirección del PCA sobre el futuro del ENA ${ }^{\text {vii }}$. 


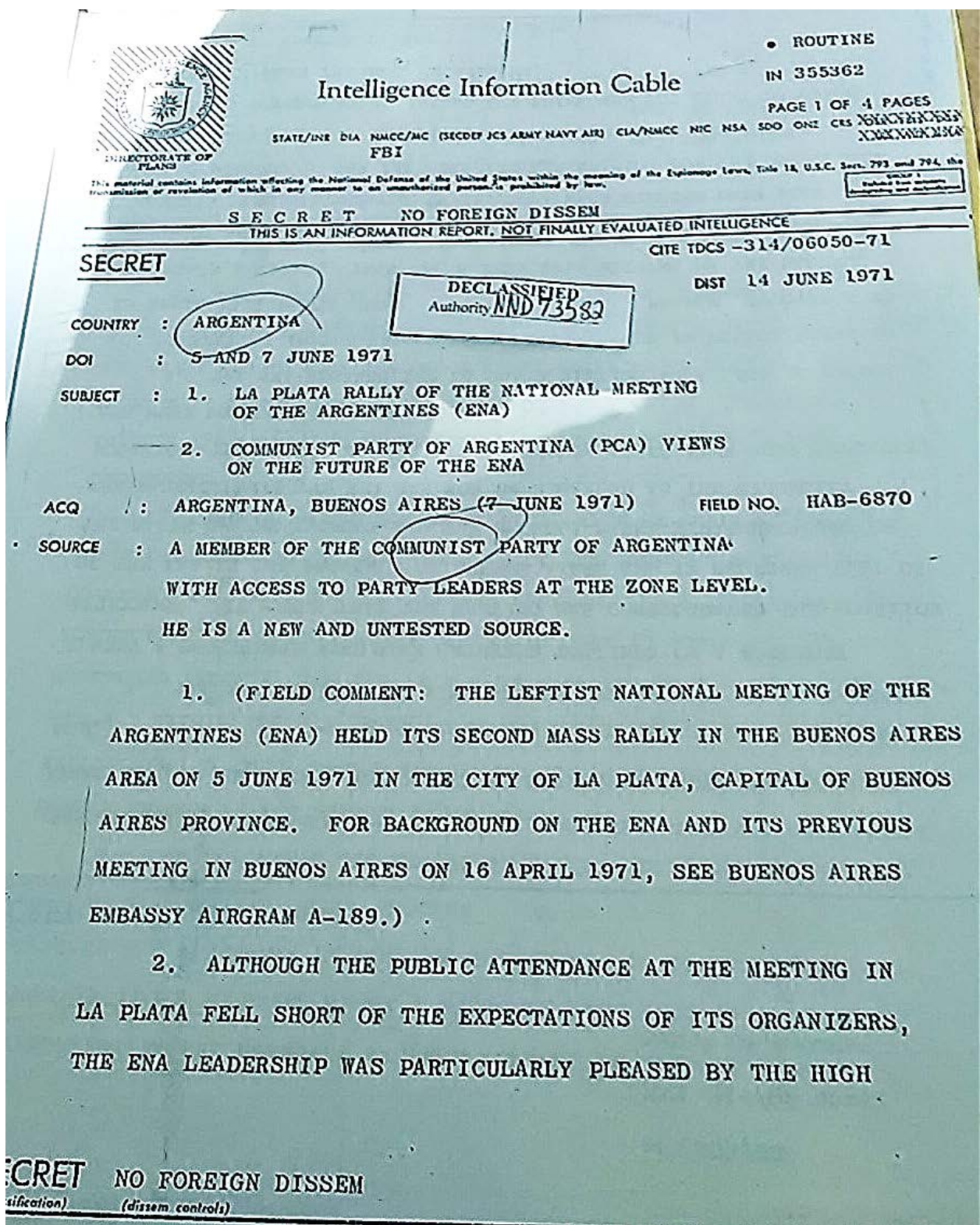

Reproducción de un cable de inteligencia en que informa que un miembro del PCA es la fuente de la información.

Fuente: Fotografía del autor

\section{5- La información disponible}

Dado que la cantidad de información sobre la Argentina disponible en los archivos de los Estados Unidos es enorme, realizar una descripción completa es imposible. Intentaremos en cambio brindar algunas ideas acerca de las áreas en la que la documentación 
norteamericana resulta particularmente útil, ya que aporta elementos que no se encuentran -o cuyo acceso resulta muy dificultoso- en repositorios de otros países.

Una de ellas es la documentación que permite echar luz sobre los procesos de toma de decisión de políticas estadounidenses hacia la Argentina, a través del análisis de las posiciones de las distintas agencias y actores involucrados, el estudio de sus estrategias y sus interlocuciones con personas e instituciones argentinas. Esto permite considerar los niveles de intervención norteamericana en la vida pública argentina a lo largo del siglo XX -pero sobre todo a partir de la segunda Guerra Mundial-, sus estrategias de presión o acompañamiento, los modos de articulación de sus intereses, las maneras en que buscaron influir sobre los partidos políticos, las elites militares, las dirigencias sociales o la prensa y las respuestas de estos actores.

Otra área en la que la información de los archivos norteamericanos es fundamental es en la propia de los análisis políticos y sociales formulados por los diplomáticos y miembros de los servicios de inteligencia norteamericanos. Aunque como resulta evidente se debe abordar estas fuentes con las precauciones metodológicas que supone considerar la perspectiva política e institucional de sus actores, estos análisis -que hemos encontrado con más frecuencia para las etapas anteriores a 1976 que para las posteriores- brindan una información muy útil para la comprensión de las prácticas de los gobiernos argentinos y de la vida política y social del país.

La densa red de informantes de la embajada norteamericana le permitía al Departamento de Estado construir información, hoy disponible, sobre la vida interna de los partidos políticos en las distintas dictaduras, brindando así una información a la que resulta casi imposible acceder en fuentes argentinas. Asimismo, el anticomunismo y las perspectivas propias de la Guerra Fría explican que se haya acumulado información -sin duda tendenciosa pero no por ello inútil- sobre los partidos de izquierda y las organizaciones sociales de esa orientación -desde grupos estudiantiles hasta sindicatos- así como sobre grupos e iniciativas anticomunistas.

Para terminar esta enumeración, y sin descartar que existan muchas otras áreas en las que la documentación norteamericana puede resultar imprescindible, no podemos dejar de referirnos a la importancia de estas fuentes para investigar sobre la represión desplegada desde los últimos años del tercer gobierno peronista y a lo largo de la última dictadura militar, 
sus ejecutores, sus víctimas, y una multiplicidad de actores que por diversos motivos se vincularon con el gobierno norteamericano.

En un balance global, los archivos públicos norteamericanos resultan un repositorio muy útil que contienen información que permite completar o complementar la disponible en los repositorios argentinos. La puesta en disponibilidad en línea de parte de estas fuentes permitirá un acceso mucho más sencillo para los investigadores argentinos, que podrán de ese modo aprovechar una fuente de información amplia y relevante.

\section{6- Referencias}

KORNBLUH, P. Pinochet. Los archivos secretos. Barcelona: Crítica, 2004.

\section{Sobre el autor}

Daniel Lvovich. Doctor en Historia por la Universidad Nacional de La Plata (Argentina). Se desempeña como Investigador docente de la Universidad Nacional de General Sarmiento y como Investigador del Consejo Nacional de Investigaciones Científicas y Técnicas (CONICET). Ha dictado cursos de posgrado en una decena de universidades de Argentina, Brasil, España y Francia. Sus investigaciones se han dedicado a diversos aspectos de la historia política y social del siglo XX.

\section{Notas}

\footnotetext{
${ }^{i}$ La OSS, Office of Strategic Services u Oficina de Servicios Estratégicos, fue el servicio de inteligencia de los Estados Unidos durante la Segunda Guerra Mundial, antecesora de la Agencia Central de Inteligencia o CIA.

ii The part of maximalism in the disturbances of january 9,10 and 11th, in Buenos Aires and other Places of the Argentine Republic, Enclosure 1 in Despatch $N^{\circ} 7421$, del Embajador Frederic Stimson al Departamento de Estado, 13 d enero de 1919, en: NARA II, Records of the Dept. of state re to internal affairs of Argentina, 1910 1929. 835.00/66-216, microcopy $\mathrm{N}^{\circ}$ 514, Roll 3.

iii Telegramas de los días 13 y 27 de enero de 1919, en NARA II, Idem.

iv Biblioteca del Congreso de los Estados Unidos. Fondo Presidente Woodrow Wilson. Rollo de microfilm 471. Partes de inteligencia. Telegrama 1357 del 22 de enero de 1919.

${ }^{v}$ Biblioteca del Congreso de los Estados Unidos. Fondo Presidente Woodrow Wilson. Rollo de microfilm 469, Weekly Inteligence Sumary 91, 22 de febrero de 1919.

${ }^{\text {vi }}$ NARA II, Country files for Argentina, 1953-1971, NAID 54977579, Caja 20, Carpeta Argentina-Poliltics.

vii Idem.
}

\begin{tabular}{l|l} 
Revista RBBA & Revista Binacional Brasil Argentina
\end{tabular} 